\title{
Evaluación de bibliotecas nacionales: elaboración de una normativa internacional y su aplicación en la Biblioteca Nacional de España
}

\author{
Por Marta De-la-Mano-González y Beatriz Albelda-Esteban
}

Resumen: Se aborda la reciente incorporación de las bibliotecas nacionales a la cultura de la evaluación de calidad mediante indicadores de rendimiento. Se explica desde su génesis el proyecto de elaboración del Informe Técnico ISO/TR 28118 , sobre indicadores de rendimiento para bibliotecas nacionales, como normativa específica común que permite la realización de benchmarking entre instituciones semejantes a nivel internacional. Se describe la implicación de los organismos internacionales IFLA, ISO y CENL en esta iniciativa, a partir de la observación de las realidades comunes a las bibliotecas nacionales. Se detalla el proceso técnico de elaboración del informe, su estructura y contenido. Se recoge la experiencia reciente de la Biblioteca Nacional de España de incorporación de indicadores de rendimiento a sus procesos de evaluación y de aplicación de los indicadores del informe ISO.

Palabras clave: Indicadores de rendimiento, Medida del rendimiento, Normalización, Evaluación de la calidad, Benchmarking, Bibliotecas nacionales, Biblioteca Nacional de España.

Title: Evaluation of national libraries: creation of an international standard and its application to the National Library of Spain

Abstract: National libraries have recently adopted a quality assessment culture using performance indicators. The project for devising Technical Report ISO/TR 28118 regarding performance indicators for national libraries is explained from its beginnings. This is a specific common standard that permit benchmarking among similar institutions at the international level. The involvement of the international bodies IFLA, ISO and CENL in this initiative is described based on observation of the situations common to all national libraries. The technical process of drawing up the report is likewise described, along with its structure and contents. Also dealt with is the recent experience of the National Library of Spain in incorporating performance indicators to its evaluation processes and applying the ISO report indicators.

Keywords: Performance indicators, Performance measures, Standardization, Quality measurement, Benchmarking, National Libraries, National Library of Spain.

De-la-Mano-González, Marta; Albelda-Esteban, Beatriz. "Evaluación de bibliotecas nacionales: elaboración de una normativa internacional y su aplicación en la Biblioteca Nacional de España". El profesional de la información, 2009, noviembre-diciembre, v. 18, n. 6, pp. 660-668.

DOI: 10.3145/epi.2009.nov.10

1. Introducción: las bibliotecas nacionales ante el reto de la evaluación

"ES HORA DE QUE LAS BIBLIOTECAS NACIONALES, como colectivo, dejen a un lado sus reservas para ponerse de acuerdo y utilizar un conjunto coherente de indicadores públicos que puedan utilizarse con fines de comparación".

Estas palabras de Line (2001, pp. 69) parecen haber marcado la actuación de las bibliotecas nacionales (BNs) en el campo de la evaluación durante los últimos años. Aunque la posición privilegiada que suelen tener en sus respectivos países las ha mantenido ajenas a la preocupación por justificar la efica- 
cia de sus procesos y la calidad de sus servicios que, desde la década de los 90 ha abocado a las bibliotecas públicas y universitarias a evaluar su rendimiento, el panorama actual es muy diferente.

\section{"A las BNs les resulta difícil seleccionar indicadores para valorar adecuadamente sus procesos y servicios"}

La iniciativa promovida por la CENL (Conference of European National Libraries) en 2001 de crear un grupo de trabajo con el fin de conocer cuál era el estado de la cuestión en este tipo de bibliotecas en lo que a medida del rendimiento se refería (Ambrožič́; Jakac-Bizjak; Peċko Mlekuš, 2003) supuso un primer y necesario acercamiento al tema. A pesar de que los datos extraídos de este trabajo no pueden generalizarse, tal como constatan sus autores, sí presentan un panorama significativo de cuál era la situación de la evaluación en una parte importante de las bibliotecas nacionales europeas en los comienzos del siglo XXI: conciencia clara de la importancia de la planificación estratégica y de la valoración del rendimiento, pero escasa vinculación práctica entre ambas funciones; conocimiento y uso de las normas internacionales vigentes para elaborar sus estadísticas e indicadores, pero poca aplicación real de estas medidas estandarizadas en los procesos de evaluación; y una aplicación sistemática de esta última mucho menos habitual de lo que las propias bibliotecas declaraban. De hecho, parece haber habido unanimidad entre las bibliotecas participantes a la hora de reconocer la necesidad de valorar sus funciones principales con regularidad.
La publicación de estos resultados en la 69 Conferencia General de la IFLA celebrada en Berlín en 2003, impulsó el movimiento de preocupación por la medida del rendimiento y la calidad que había empezado a gestarse en los organismos internacionales vinculados a las BNs, los cuales situaron la evaluación entre sus prioridades de actuación.

Tanto la CENL como la Sección de Bibliotecas Nacionales de la IFLA comparten en la actualidad un objetivo común incluido en sus respectivos planes estratégicos": "servir de foro a las BNs para intercambiar experiencias con otras BNs, para promover el benchmarking, las buenas prácticas y los modelos de calidad, y mejorar las técnicas de gestión". Al amparo de este objetivo, se han planteado en los últimos años una serie de acciones orientadas a la difusión y uso de medidas del rendimiento y valoración de la calidad en las BNs que van, desde la creación o reactivación de grupos de trabajo sobre evaluación hasta la realización de encuestas sobre indicadores de actividad, pasando por la organización de encuentros y simposios para el intercambio de opiniones y experiencias sobre este aspecto.

Es en este contexto donde se ha gestado el proyecto que a continuación presentamos: la elaboración de una normativa internacional específica sobre el uso de indicadores de rendimiento para bibliotecas nacionales.

\section{Necesidad de una normativa específica: Informe Técnico ISO/TR 28118}

\subsection{Origen y evolución del pro- yecto}

Las BNs son instituciones únicas en sus países y sus datos no se pueden comparar con facilidad, puesto que su misión y funciones difieren de unos países a otros, no tienen un grupo de usuarios específicos, y realizan tareas que las distinguen de otros tipos de bibliotecas como la colección y preservación de la herencia cultural nacional, la publicación de la bibliografía nacional, y el papel director en la cooperación internacional (ISO, 2009 , p. v). Estos factores determinan que les resulte difícil identificar y seleccionar indicadores que sean adecuados para la valoración de sus procesos y servicios, incluso en el caso de aquéllos establecidos por la normativa internacional, o recogidos en manuales ampliamente difundidos.

Esta realidad se puso de manifiesto en el Congreso de la IFLA de 2005 celebrado en Oslo, donde se constató la necesidad de establecer indicadores de rendimiento específicos, ya que los propuestos en la norma ISO 11620, en ese momento en proceso de revisión, no resultaban adecuados para la evaluación de este tipo de bibliotecas dadas sus especiales características (Poll, 2008, p. 111).

\section{"El principal criterio adoptado por el grupo de trabajo en la selección de los indicadores ha sido el de su utilidad"}

Trasladada a la Organización Internacional de Normalización (ISO), el Subcomité 8 del Comité 46 de esta organización ${ }^{2}$-especializado en estadística y evaluación del rendimiento-, tomó la decisión ${ }^{3}$ de poner en marcha un nuevo proyecto destinado a elaborar un Informe Técnico sobre indicadores de rendimiento para BNs. Se optó por la elaboración de este tipo de documento, de carácter informativo, debido a que una parte destacada de los indicadores propuestos habían sido diseñados por primera vez y se 
pretendía que, antes de su incorporación a una norma internacional, fueran aplicados para comprobar su utilidad y viabilidad reales.

Con esta finalidad, y una vez aprobada la iniciativa, se creó en enero de 2007 un nuevo grupo de trabajo sobre "Medidas de calidad para bibliotecas nacionales", coordinado por Roswitha Poll e integrado por 12 miembros procedentes de 11 países diferentes, entre ellos España ${ }^{5}$. Se tomó como punto de partida para la elaboración del Informe Técnico una propuesta de indicadores (Poll; Jönsson-Adrial, 2006) que había sido presentada y debatida previamente ante el Comité Permanente de la Sección de Bibliotecas Nacionales de la IFLA, durante la Conferencia General celebrada en Seúl en agosto de 2006 (IFLA, 2006). Desde ese momento y hasta su culminación, el proyecto ha contado con la colaboración y el respaldo de la IFLA, así como de la $C E N L^{6}$.

Aproximadamente un año después de su creación el grupo de trabajo tenía preparado un borrador del Informe Técnico ISO/TR 28118 que sometió a la votación de los países miembros, con resultado positivo $^{7}$. No obstante, muchos de estos países enviaron comentarios y observaciones sobre el contenido del documento que fueron debatidos $\mathrm{y}$, en su caso, incorporados al texto definitivo del Informe Técnico, que se publicó el 5 de mayo de 2009.

\subsection{Selección y diseño de los in- dicadores}

El principal criterio adoptado por el grupo de trabajo en la selección de los indicadores ha sido el de su utilidad, eligiéndose los que se consideraron más valiosos para la valoración de las principales funciones que desempeñan las bibliotecas nacionales (ISO, 2009, pp. 11). Para poder aplicarlo, y dada la variedad que caracteriza a estas instituciones, previamente se elaboró una formulación general de la misión, objetivos y funciones propias de este tipo de bibliotecas, a partir de los puntos en común identificados en las declaraciones incluidas en los sitios web de varias BNs (Poll, 2008, pp. 112).

Delimitadas las principales áreas de actuación, el siguiente paso fue identificar y definir los indicadores de rendimiento más adecuados para cada una de ellas. Además del criterio ya señalado de su utilidad, se tuvo en cuenta que aportaran información sobre la calidad, y no tanto la cantidad, del servicio. Se descartaron los indicadores de resultado e impacto, a pesar de su interés a la hora de valorar la proyección real de la biblioteca, por considerar que este tipo de instrumentos no están todavía suficientemente consolidados.

\section{"La incorporación de ejemplos de los resultados obtenidos en el uso del indicador es uno de los elementos más novedosos del Informe Técnico"}

En el proceso de selección y diseño de los indicadores se han utilizado diferentes fuentes. Se tomó como principal referencia la última edición de la norma internacional ISO 11620:2008 (ISO, 2008) sobre indicadores de rendimiento para bibliotecas, escogiendo aquéllos que pudieran servir a los cometidos de las BNs. Se eligió un total de 15 relacionados con aspectos como el rendimiento de los procesos técnicos, el acceso de los usuarios a los servicios, y el aprovechamiento de los potenciales de la biblioteca, comunes al funcionamiento de cualquier institución bibliotecaria, aunque adaptándolos a los requisitos y condiciones de las bibliotecas nacionales.
El resto de los indicadores propuestos, otros 15 , cubren actividades propias del funcionamiento de este tipo de bibliotecas y, por tanto, no se contemplan en la citada norma internacional. En este caso, o bien se diseñaron indicadores nuevos como los vinculados a la preservación, la digitalización o la cooperación internacional; o bien se incorporaron otros cuyo uso se hubiera generalizado entre las bibliotecas nacionales, como la rapidez en el servicio de referencia. La fuente principal utilizada para construir o identificar este tipo de instrumentos fueron los informes anuales y estadísticas elaboradas por las diferentes bibliotecas nacionales y disponibles a través de sus webs.

Para la descripción de cada uno de los indicadores incluidos en el Informe Técnico se ha adoptado un esquema que difiere en algunos aspectos del utilizado en la norma ISO 11620:20089. Concretamente, esta descripción incluye los siguientes elementos:

- Nombre: utilizado para identificar de forma inequívoca al indicador.

- Contexto: sitúa el uso y la importancia del indicador en el entorno actual de funcionamiento de las BNs.

- Definición: describe su naturaleza, especificando los datos que incluye y su relación.

- Objetivos del indicador: explica lo que pretende medir en relación a las funciones y objetivos de la biblioteca; los beneficios que se derivan de su uso; y las limitaciones en su aplicación.

- Método: describe el modo en que se obtienen los datos y se calculan los resultados.

- Interpretación y uso de los resultados: presenta las conclusiones que se pueden extraer de los resultados del indicador, así como las dificultades y circunstancias que pueden afectar a estos resultados. 
- Ejemplos y lecturas adicionales: proporciona referencias que documentan la fuente de la que se ha tomado el indicador $u$ otros indicadores similares. Siempre que ha sido posible se han incorporado, además, ejemplos de los resultados obtenidos en el uso del indicador para ayudar a las bibliotecas a interpretar sus propios resultados.

\section{"Los indicadores de rendimiento han de valorar: la cobertura de la colección, la accesibilidad de los recursos, el rendimiento de los servicios, las condiciones de preservación y el grado de cooperación"}

Este último elemento es el más novedoso y creemos que resulta de gran interés y utilidad, ya que incluye experiencias reales sobre el uso del indicador concreto de que se trate, llevadas a cabo preferentemente, pero no exclusivamente, en bibliotecas nacionales. La búsqueda de ejemplos y experiencias del uso real de cada indicador ha representado la tarea más difícil de llevar a cabo, bien porque tales indicadores realmente no se utilizaban o bien porque, aun utilizándose no se habían dado a conocer los resultados obtenidos con su aplicación. En este sentido cabe destacar el papel desempeñado por algunas de las bibliotecas nacionales participantes en el grupo de trabajo que han hecho un gran esfuerzo por probar algunos de los indicadores propuestos, como la Diet National Library de Japón, las alemanas, y la Biblioteca Nacional de España.

\subsection{Estructura del Informe Téc- nico}

A la hora de abordar este aspecto, cabe distinguir dos niveles de análisis: la estructura formal y la estructura interna del documento.

La primera es muy similar a la de las normas e informes internacionales sobre medida del rendimiento que le han precedido: la norma $I S O$ 11620:2008 y el Informe Técnico 20983:2003 sobre indicadores de rendimiento para servicios bibliotecarios electrónicos. Se articula en las siguientes partes:

- La introducción, que justifica su necesidad y su interés en el contexto actual de medida del rendimiento en las BNs.

- Un marco conceptual, integrado por seis apartados, que aborda los principales aspectos relacionados con el alcance, el uso, y la presentación de los indicadores seleccionados, y que constituye el núcleo del informe. Destaca aquí la incorporación de un glosario que incluye la definición de los principales términos especializados utilizados en el texto, para facilitar su comprensión. De los 56 vocablos que contiene tan sólo 4 son originales de este documento; el resto han sido extraídos o adaptados de los vocabularios incluidos en otras normas: la ISO 2789:2006 sobre estadística bibliotecaria, la ISO 5127:2001 sobre vocabulario, la ISO 9707:2008 sobre estadísticas relacionadas con la producción y distribución de libros, periódicos, publicaciones periódicas y publicaciones electrónicas, y la citada ISO 11620:2008.

A continuación se incluyen tres anexos de carácter informativo.

- Un primer anexo en el que se describen individual y detalladamente cada uno de los indicadores enumerados en la parte anterior y que, pese al carácter auxiliar que se asocia a este tipo de apartado, representa una parte esencial del Informe Técnico, puesto que despliega la propuesta de indicadores inicialmente planteada.

- Un segundo anexo en el que se incluyen la misión y los objeti- vos formulados para las bibliotecas nacionales, ya mencionados.

- Un tercero que proporciona una visión general de los métodos y los aspectos de calidad que deben tenerse en cuenta a la hora de incorporar al fondo los recursos de internet.

- Finalmente, una bibliografía especializada sobre el tema del Informe Técnico.

Más interesante resulta el análisis de la estructura interna. Presenta un diseño vertical que parte de la formulación general de la misión, y se despliega en niveles sucesivos con un grado creciente de concreción y operatividad que incluyen la formulación de los fines y funciones, la identificación de las actividades y servicios orientados al logro de los objetivos y finalmente, la propuesta de los indicadores de rendimiento que van a medir la eficacia, eficiencia y calidad de los resultados obtenidos en el ejercicio de dichas actividades.

\section{"Además de ser un instrumento para la gestión de la calidad, los indicadores proporcionan bases para la comparación con instituciones semejantes a nivel internacional"}

La misión se define en los siguientes términos, con una redacción similar a la elaborada por la Sección de Bibliotecas Nacionales de la IFLA: "Las bibliotecas nacionales tienen responsabilidades especiales, a menudo establecidas por ley, sobre la herencia cultural de un país. Recogen y preservan la herencia documental nacional y proporcionan y aseguran acceso permanente al conocimiento y a la cultura del pasado y del presente. Ofrecen 
servicios centrales y asumen un papel de liderazgo en el sector de las bibliotecas y de la información" (ISO, 2009, pp. 78).

A partir de aquí se han establecido seis áreas principales en el funcionamiento de estas bibliotecas (colección, acceso, marketing, preservación, cooperación y gestión) y un área adicional que recoge otras funciones que pueden desempeñar, para cada una de las cuales se ha formulado un número determinado de objetivos. Delimitados los objetivos, se han identificado diversos servicios y actividades orientadas a su consecución, y se ha planteado una serie de indicadores para su valoración. Las actividades e indicadores seleccionados han sido los siguientes:

1. Construcción de la colección nacional. Incluye dos indicadores sobre la cobertura de la colección nacional: porcentaje de publicaciones nacionales adquiridas por la $\mathrm{BN}$ y porcentaje de los títulos impresos nacionales solicitados que posee la colección.

2. Accesibilidad de los servicios. Este aspecto se divide en cuatro áreas:

- Catalogación: contiene dos indicadores para evaluar la incorporación de nuevos registros a la bibliografía nacional y el acceso a los materiales especiales que posee la biblioteca: porcentaje de nuevas entradas en la bibliografía nacional $\mathrm{y}$ de materiales raros accesibles a través de los catálogos web.

- Rapidez y facilidad de acceso: recoge varios indicadores planteados esencialmente para medir el tiempo que se emplea en la realización de determinados procesos o servicios, y la facilidad de su uso: tiempo medio de procesamiento de los documentos, correcta colocación de los materiales en las estanterías, tiempo medio de recuperación de documentos de depósitos cerrados, rapidez del préstamo interbibliotecario, y acceso directo desde su web.

- Uso: es uno de los aspectos que, junto con el anterior, posee un mayor número de indicadores, orientados a valorar el uso que reciben determinados productos y servicios de las bibliotecas nacionales, así como la satisfacción del usuario con la prestación de los mismos: uso de publicaciones periódicas adquiridas durante los últimos tres años, coste por descarga de documento electrónico, tasa de ocupación de asientos, número de asistencias por acontecimiento cultural, y satisfacción de los usuarios.

- Digitalización: contiene tres indicadores que valoran las actividades de digitalización de la biblioteca: número de documentos digitalizados por 1000 títulos de la colección, porcentaje de documentos digitalizados por colección especial, número de unidades de contenido descargados por documento digitalizado.

3. Oferta de los servicios de referencia. Comprende indicadores diseñados para evaluar dos aspectos esenciales en la prestación de este tipo de servicio como son la eficacia y la agilidad en la respuesta: tasa de respuestas correctas y rapidez de las operaciones de referencia.

4. Generación de potenciales para el crecimiento. Los indicadores que se incluyen en este apartado pretenden medir la capacidad de la biblioteca para aprovechar e incrementar los potenciales que posee en cuanto a medios humanos y económicos: porcentaje del personal de la biblioteca que proporciona servicios electrónicos, número de horas de asistencia a cursos de formación reglada por miembro de la plantilla, porcentaje de los ingresos de la biblioteca recibidos por subvención especial o renta generada, porcentaje de la plantilla en proyectos de cooperación nacional e internacional.
5. Preservación de la colección. Abarca indicadores destinados a valorar las condiciones de conservación y preservación en que se encuentran los materiales de la biblioteca: porcentaje de la colección en condiciones estables, porcentaje de materiales que necesitan tratamiento de conservación/restauración que reciben este tratamiento, porcentaje de la colección almacenado en un entorno adecuado.

6. Gestión eficiente. Los indicadores incluidos en este último apartado están relacionados con el uso apropiado de los medios humanos y económicos (eficiencia) en la ejecución de los procesos y servicios de la biblioteca: costes de personal por título catalogado, costes de personal por préstamo, productividad de los trabajadores en el procesamiento de los materiales, productividad de los trabajadores en los servicios de préstamo y entrega de documentos.

\section{“EI TR 28118 de ISO ha servido como marco conceptual para el diseño de la serie de indicadores de la BNE"}

La representación gráfica de la estructura interna del informe sería la del gráfico $1^{10}$ :

La importancia real de estas actividades en el trabajo de la biblioteca depende de sus circunstancias, por lo que el Informe Técnico indica que son las propias bibliotecas nacionales, de acuerdo con las autoridades que las gobiernan, las instituciones que las financian y sus propios usuarios, las que deben elegir qué indicadores resultan más adecuados a su situación concreta (ISO, 2009, pp. 11). No obstante, al margen de sus prioridades y características particulares, y a la luz de 


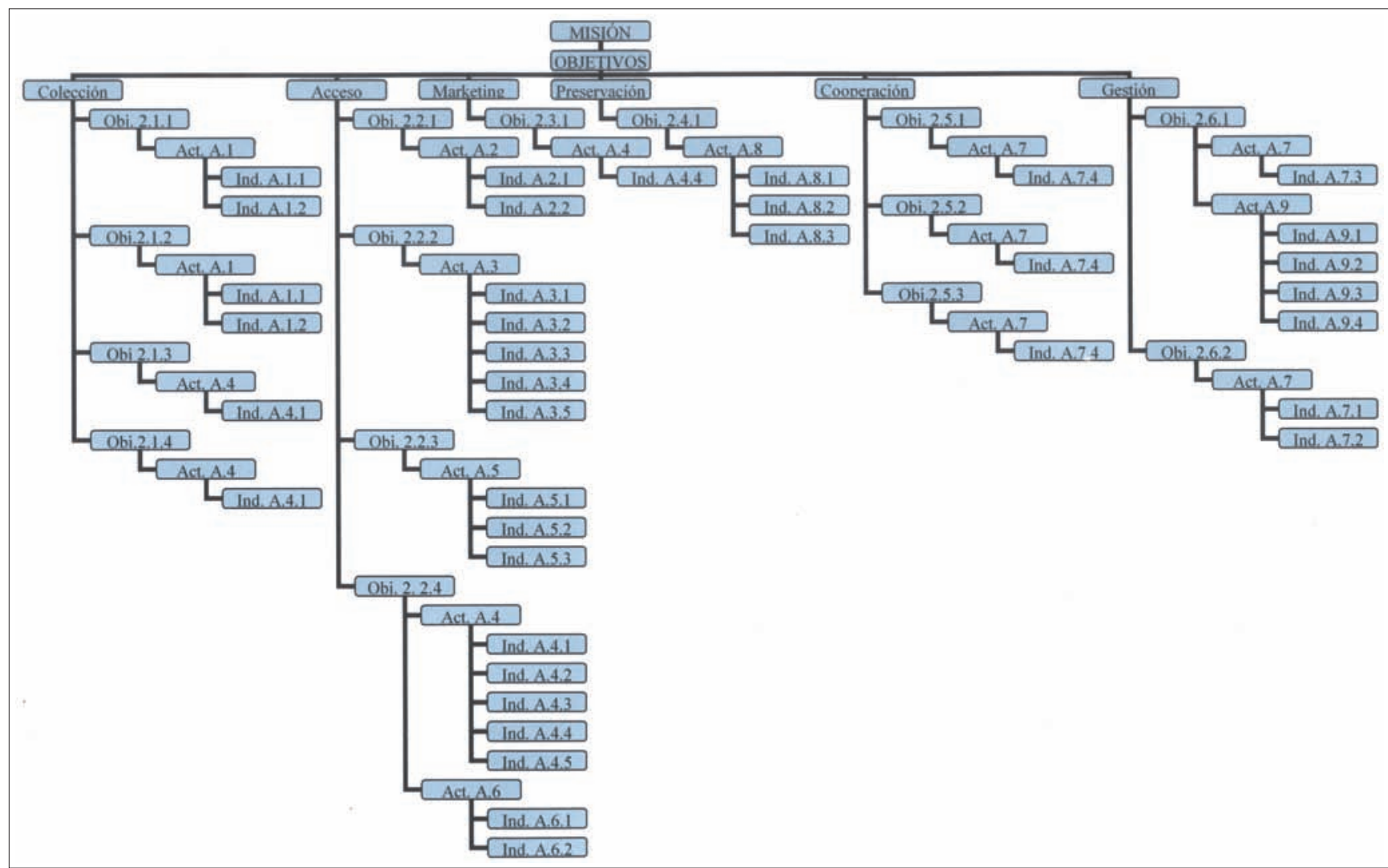

Gráfico 1. Relaciones entre misión, objetivos, actividades e indicadores en el informe ISO/TR 28118

la misión general formulada, los indicadores de rendimiento utilizados por una BN deberían hacer posible valorar, al menos, los siguientes aspectos: cobertura de la colección nacional, accesibilidad de los recursos, rendimiento de los servicios, condiciones de preservación, y grado de cooperación nacional e internacional de la biblioteca.

A continuación presentamos el caso de la aplicación de esta normativa internacional a la Biblioteca Nacional de España (en adelante $B N E$ ), que ha colaborado en la elaboración del Informe Técnico y está siendo pionera en su utilización.

\section{Medida del rendimiento en la BNE: aplicación del Informe Técnico ISO/TR 28118:2009}

3.1 Evaluación mediante indicadores: situación de partida

La $B N E$ no ha vivido un proceso de evaluación de calidad como las bibliotecas universitarias. El punto de partida de ese proceso hay que situarlo en la paulatina incorporación de las líneas impulsadas por el Ministerio de Administraciones Públicas desde su Marco General para la Mejora de la Calidad en la Administración General del Estado.

Las primeras iniciativas se remontan a los años 1986-87, cuando se hicieron las primeras encuestas de satisfacción a los usuarios en las salas de lectura, y al período 20002005, cuando se elaboraron encuestas de evaluación de servicios. Otro hito fundamental fue la aprobación en 2003, de la Carta de Servicios en la que ya se requería la utilización de unos indicadores para medir el cumplimiento de los compromisos de calidad contraídos con los ciudadanos.

A partir de 2005 la $B N E$ empezó a elaborar su primer Plan Estratégico para el período 2006-2008, que exigía el establecimiento de unos fines evaluables mediante indicadores.

Por tanto, la experiencia de trabajo con indicadores de rendimien- to es muy reciente en el caso de la $B N E$.

En mayo de 2006 la institución asistió como observadora al Grupo de Trabajo 7 del Comité Técnico 46 de la ISO sobre Medidas de Calidad para Bibliotecas Nacionales. Desde entonces se ha venido colaborando con este comité en la revisión del borrador del Informe Técnico sobre indicadores de rendimiento para bibliotecas nacionales.

Este borrador se fundamentaba sobre experiencias de instituciones que llevaban años trabajando con indicadores propios. Se vio entonces el interés que tendría ensayar los nuevos indicadores en una biblioteca como la española, donde no se partía de ninguna experiencia previa y donde existía la voluntad de incorporar indicadores de rendimiento para valorar el cumplimiento de sus objetivos.

Desde el primer momento la $B N E$ fue receptiva a la propuesta de colaboración de la ISO. Además de ser un instrumento para la ges- 
tión de la calidad, los indicadores proporcionarían unas bases para la comparación con instituciones semejantes a nivel internacional.

El trabajo con los nuevos indicadores empezó a ponerse en marcha a finales de 2007 y se ha realizado a lo largo de todo 2008. En este tiempo se ha trabajado a dos niveles: se ha comprobado la adecuación de los indicadores del borrador ISO a la realidad de nuestra Biblioteca Nacional y se ha trabajado en la elaboración de indicadores ajustados a la medición de los objetivos concretos del plan estratégico anual.

\subsection{Experiencia de implantación}

Los trabajos previos al diseño de los nuevos indicadores consistieron en la realización de un análisis comparativo entre los del borrador ISO y los datos estadísticos que la $B N E$ recogía para uso interno. Era necesario coordinar los datos que ya se recogían habitualmente y de manera independiente en los distintos departamentos y unidades de gestión de la $B N E$.

Para todo ello se creó un primer grupo de trabajo integrado por personas de distintos departamentos y servicios con experiencia en la recogida de datos estadísticos mensuales y conocedores de los procedimientos internos.

En el primer trimestre de 2008 se realizó un trabajo de consenso e información a nivel departamental y se fijó el plan de trabajo y el cronograma para la recogida de datos de 2008, a modo de primer ensayo previo a la implantación definitiva prevista para 2009.

\section{Diseño de los indicadores}

El TR 28118 de ISO ha servido como marco conceptual para el diseño de la serie de indicadores de la $B N E$.

Primero hubo que hacer una adaptación de sus contenidos a la propia realidad de la $B N E$, compro- bando si la metodología de cálculo propuesta podía seguirse teniendo en cuenta nuestros datos fuente $\mathrm{y}$ nuestros propios flujos de trabajo.

En la mayor parte de los casos ha sido posible calcular el indicador ISO pero con adaptaciones en la metodología. En la medida de lo posible se ha preferido utilizar datos extraídos de fuentes automatizadas, que no siempre se ajustan a los métodos de cálculo manuales utilizados en el informe ISO.

\section{Tipos de indicadores}

El cuadro de indicadores de evaluación de la $B N E$ se compone de cuatro bloques que recogen un total de 73. Cada bloque comprende un área diferente que aglutina una serie de indicadores cuantitativos y cualitativos. Estos bloques son:

1. Indicadores para evaluar colecciones: comprende 25 agrupados en 5 subgrupos:

- Cobertura: a partir de datos de ingreso por Depósito Legal, se evalúa su impacto en la formación de la colección bibliográfica nacional.

- Accesibilidad: se evalúa la disponibilidad de las colecciones, su difusión a través de catálogos automatizados y de proyectos de digitalización y la normalización de canjes y donativos

- Conservación: se evalúa la adecuación e impacto de las actuaciones y planes de preservación.

- Uso: se evalúa el grado de circulación de las colecciones y la adecuación de las mismas según la demanda de los usuarios.

- Difusión de buenas prácticas relacionadas con la formación de usuarios en colecciones electrónicas y preservación documental.

2. Indicadores para evaluar servicios que propone la $B N E$, agrupados a su vez en tres subgrupos:

- Tiempos de respuesta en servicios: se mide la rapidez en la resolución de preguntas de información referencial, en el servicio de fondos $\mathrm{y}$ en suministro de documentos y préstamo interbibliotecario.

- Acceso a nuevos servicios de búsqueda e información: se mide la tasa de uso de nuevos servicios de información recientemente implantados de forma remota: metabuscador, SFX, descargas de la Biblioteca Digital Hispánica.

- Uso de servicios presenciales: se mide la tasa de ocupación de los puestos de lectura de las distintas salas y la tasa de asistencia a actividades culturales. Se incluye además un bloque de indicadores que evalúan la satisfacción de usuarios.

3. Indicadores para evaluar los medios humanos: son 7 agrupados en dos bloques:

- Tiempos invertidos en flujos de trabajo: se evalúa la productividad del personal dedicado a distintos procesos y servicios, así como el coste de unidades procesadas o servidas.

- Formación del personal: se cuantifica el número de horas invertidas por el personal bibliotecario en la formación.

4. Indicadores para evaluar la proyección externa de la institución:

- Cooperación: se evalúa la presencia de la $B N E$ en proyectos de cooperación nacional e internacional.

- Eficiencia en la gestión económica: se cuantifican los ingresos económicos excepcionales.

\section{Ficha de recogida de datos de in- dicadores}

Para cada uno de los indicadores se ha elaborado una ficha donde se recoge toda la información sobre el mismo. La ficha se compone de tres bloques de información principales:

1. Descripción del indicador: recoge la información técnica resumida en los siguientes puntos: 
- Definición del indicador y su finalidad.

- Metodología de cálculo: recoge en detalle el procedimiento de cálculo.

- Problemas a detectar: son las posibles disfunciones de gestión o funcionales que puede sacar a la luz el indicador.

- Decisiones de gestión que podemos tomar: posibles actuaciones que se pueden llevar a cabo a la luz de los resultados del indicador.

- Fuentes de donde se recogen los datos estadísticos originales necesarios para el cálculo del indicador.

- Fecha comienzo de aplicación del indicador: de cara a la continuidad en el tiempo, es necesario recoger la fecha en la que se empezó a utilizar.

- Periodicidad de cálculo del indicador: dependerá en cada caso de factores como la dificultad y el coste de cálculo. No siempre es posible medir determinados datos anualmente, debido al elevado coste de la recogida de datos y a que la información obtenida no suele variar sustancialmente de un año a otro.

- Fecha de recogida del indicador: la experiencia nos indica cuándo es el momento del año en el que se debe realizar. En algunos casos es posible calcularlo a partir de una muestra representativa de datos del primer semestre del año. En la mayor parte de los indicadores es preciso finalizar el año en curso para realizar el cálculo partiendo de cifras anuales. Otra opción es utilizar datos semestrales y hacer una estimación anual.

- Responsables de los datos del indicador: son las personas que se harán responsables de la recogida de datos y su cálculo, así como de vigilar las modificaciones que pueda haber en las fuentes y en la naturaleza de los datos originales.
- Validación: es la persona responsable del diseño técnico del indicador.

- Fecha de validación: es la fecha en la que se ha validado su adecuación y robustez.

- Referencia ISO: se indica en cada caso si el indicador equivale a alguno de los recogidos en el ISO/ TR 28118.

2. Actuaciones ligadas a la implantación de los indicadores: Está previsto recoger aquí el objetivo estratégico anual, así como las actuaciones y fines operacionales más concretos vinculados a cada uno. A finales de 2009 la BNE llevará a cabo la primera evaluación de la implantación de indicadores aplicados a medir el cumplimiento de fines estratégicos.

\section{Seguimiento del indicador:}

- Responsable de la medición: puede coincidir o no con el responsable del dato del indicador.

- Valor a alcanzar y plazos de consecución: se concretan en los objetivos operacionales del plan estratégico. El indicador expresa en qué medida los resultados se han acercado al objetivo fijado inicialmente.

- Valores de referencia internos: si se cuenta con datos de años anteriores.

- Valores de referencia externos: son datos de instituciones semejantes. Facilitan la evaluación comparativa. Son difíciles de obtener ya que no siempre estos datos de gestión interna llegan a publicarse.

- Posibles desviaciones: son las variaciones respecto del valor objetivo.

- Justificación de las desviaciones: explica las posibles incidencias habidas y que han impedido la consecución de un determinado fin.

- Acciones de mejora para corregir las desviaciones: son las decisiones que se tomarán para la consecución del objetivo.

\section{Conclusiones}

2008 ha sido el primer año de trabajo con indicadores de rendimiento a modo de ensayo en la $B N E$. La principal dificultad ha sido la recogida de datos, debido a que la $B N E$ estaba viviendo paralelamente su primer año tras la migración del catálogo. Por otra parte ha habido que contar con la no automatización de algunos procesos y la dispersión y escasa fiabilidad de algunas fuentes de datos. A ello hay que sumar la falta de experiencia en la obtención de algunos indicadores.

No obstante, a pesar de las dificultades, el esfuerzo ha servido como aprendizaje para la implantación prevista para 2009. Tras la obtención de los primeros indicadores, se ha elaborado un primer informe de evaluación sobre los objetivos 2008. Por primera vez la $B N E$ ha realizado una valoración global de su actividad que constituye una base para consolidar en los años futuros.

\section{"Por primera vez la BNE ha realizado una valoración global de su actividad que constituye una base para consolidar en los años futuros"}

Aunque la comparación entre bibliotecas nacionales es compleja, ya que se parte de procesos y realidades distintas, podemos contar al menos con unos mismos referentes que hagan posible valorar la evolución de instituciones semejantes.

Por otra parte, la $B N E$ cuenta ya con un referente inestimable en su Plan Estratégico 2009-2011 (Biblioteca Nacional de España, 2009). 


\section{Notas}

1. En el caso de la CENL este objetivo se recoge en el plan estratégico para el período 2005-2008, y en el de la Sección de Bibliotecas Nacionales de la IFLA en el plan elaborado para el período 2007-2009.

http://www.cenl.org/docs/CENL_StrategicPlan_ 2005_08.pdf

http://archive.ifla.org/VII/s1/annual/sp01-2009. htm

2. Technical Committee ISO/TC 46, Information and documentation. Subcommittee SC 8, Quality-statistics and performance evaluation.

3. Esta decisión fue tomada en la reunión del subcomité celebrada en mayo de 2006 en París.

4. Se trata del Grupo de trabajo 7 del Subcomité 8: ISO/TC 46/SC8/WG 7 Quality measures for national libraries.

5. La representante española fue Marta De-laMano-González, nominada como experta por el Comité 50 de Aenor.

6. El responsable del grupo de trabajo en indicadores de rendimiento para bibliotecas nacionales (Working Group on Performance Evaluation for National Libraries) de la CENL, Willy Vanderpijpen, ha formado parte del grupo de trabajo ISO/TC 46/SC 8/WG 7 que ha elaborado el Informe Técnico.

7. De los países con derecho a voto, 11 fueron positivos (7 con comentarios), y hubo 3 abstenciones.

8. La última versión de la norma había sido votada unos días antes de la primera reunión del grupo de trabajo, el 15 de enero de 2007. Fue publicada en octubre de 2008.

9. La descripción del indicador no incluye algunos de los campos que incorpora la norma 11620:2008 -el alcance del indicador, las fuentes y los indicadores relacionados-, e introduce otros nuevos -el contexto y los ejemplos y lecturas adicionales- que se han adoptado de la propuesta utilizada en la segunda edición del Manual de la IFLA (Poll; Boekhorst, 2007).

10. Las actividades e indicadores incluidos en el gráfico están identificados con el código que poseen en el Informe Técnico. Para los objetivos se ha establecido un código compuesto por la numeración del área en la que se inscriben acompañada de un dígito que establece el orden correlativo en el que se encuentran dentro de dicha área.

11. Real Decreto $951 / 2005$, de 29 de julio, por el que se establece el marco general para la mejora de la calidad en la Administración General del Estado (BOE de 3 de septiembre de 2005).

12. El Plan de Modernización de las Instituciones Culturales de la Administración General del Estado aprobado en Consejo de Ministros el 7 de septiembre de 2007 recoge una serie de buenas prácticas para la mejora de la gestión de la Institución y de la calidad del servicio prestado a la ciudadanía. Entre ellas: "la transparencia en la gestión y la información periódica a la ciudadanía acerca del proyecto cultural de la Institución y sus programas de actuación, concretado en todos los aspectos detallados en el proyecto de gestión".

13. Desde 2008 la $B N E$ colabora con el Working Group on Performance Evaluation for National Libraries de la CENL en la revisión y puesta en práctica del Informe Técnico ISO/TR 28118.

14. Para el proceso de implantación se ha tenido en cuenta la norma UNE 66175 - Sistemas de gestión de la calidad. Guía para la implantación de sistemas de indicadores, de 2003.

15. Un ejemplo de esto son los indicadores que miden tiempos de respuesta en procesos. En ellos es crucial fijar los puntos en los que se van a entender el inicio y el final del proceso de una manera inequívoca y esto viene condicionado por las opciones que ofrezca el sistema de gestión utilizado.

16. Para la medición de satisfacción se ha llevado a cabo en 2009 una encuesta global a usuarios.

17. Tal es el caso del indicador de satisfacción de usuarios, que requiere de recursos humanos extraordinarios para la elaboración de una encuesta.

\section{Bibliografía}

Ambrožič, Melita; Jakac-Bizjak, Vilenka; Peċko Mlekuš, Helena. "Performance evaluation in European national libraries". En: World library and information congress, 69th IFLA General conference and council. Berlin, 2003.
http://www.ifla.org/IV/ifla69/papers/024e-Ambrozic_Jakac-Bizjak_Mlekus.pdf

Biblioteca Nacional de España. Plan estratégico 2009-2011.

http://www.bne.es/export/sites/BNWEB1/es/ LaBNE/PlanEstrategico/docs/Plan_estrategico_ 09-11def.pdf

IFLA National Libraries Section. Minutes of meetings 2006: $1^{\text {st }}$ meeting, Seoul, Korea. http://archive.ifla.org/VII/s1/annual/s1-SCminutes2006-1.pdf

ISO. ISO 11620:2008, Information and documentation-Library performance indicators. ISO, 2008.

ISO. ISO/TR 28118:2009, Information and documentation-Performance indicators for national libraries. ISO, 2009.

Line, Maurice. "How well are national libraries doing?". Alexandria, 2001, v. 13, n. 2, pp. 67-69. ISBN 978-0-754-61682-5.

Poll, Roswitha. "The cat's pyjamas? Performance indicators for national libraries". Performance measurement and metrics, 2008, v. 9, n. 2, p. 110-117. ISSN 1467-8047.

Poll, Roswitha; Boekhorst, Peter te. Measuring quality: performance measurement in libraries. Munich: Saur, 2007, ISBN 978-3-598-22033-3

Poll, Roswitha; Jönsson-Adrial, Christina Performance indicators for national libraries. a list of possible indicators, taken from the new draft of the standard ISO 11620 and from practical examples tested by national or regional libraries.

http://archive.ifla.org/VII/s1/pub/s1-PerformanceIndicators2006.pdf

Marta De-la-Mano-González. Departamento de Biblioteconomía y Documentación, Universidad de Salamanca.

lamano@usal.es

Beatriz Albelda-Esteban. Biblioteca Nacional de España. beatriz.albelda@bne.es

\section{Suscripciones}

Renovar (o comenzar) la suscripción a "El profesional de la información" es ágil y sencillo.

Usted puede gestionar online su suscripción conectándose a esta web: http://www.elprofesionaldelainformacion.com/suscripciones.htm/

Si lo desea puede comunicar con nosotros dirigiéndose a: suscripciones@elprofesionaldelainformacion.com

o al teléfono: $+34-609352954$ 